\title{
Is social class standardisation occupational studies?
}

\author{
CHANTAL BRISSON, , DANA LOOMIS*, AND NEIL PEARCE ${ }^{\dagger}$ \\ From the Department of Epidemiology, ${ }^{*}$ University of North Carolina School of Public Health, Chapel Hill, NC \\ 27701, USA, and Department of Community Health, ${ }^{\dagger}$ Wellington Clinical School of Medicine, Wellington, New \\ Zealand
}

SUMMARY Social class standardisation has been proposed as a method for separating the effects of occupation and "social" or "lifestyle" factors in epidemiological studies, by comparing workers in a particular occupation with other workers in the same social class. The validity of this method rests upon two assumptions: (1) that social factors have the same effect in all occupational groups in the same social class, and (2) that other workers in the same social class as the workers being studied are free of occupational risk factors for the disease of interest. These assumptions will not always be satisfied. In particular, the effect of occupation will be underestimated when the comparison group also has job-related exposures which cause the disease under study. Thus, although adjustment for social class may minimise bias due to social factors, it may introduce bias due to unmeasured occupational factors. This difficulty may be magnified when occupational category is used as the measure of social class. Because of this potential bias, adjustment for social class should be done only after careful consideration of the exposures and disease involved and should be based on an appropriate definition of social class. Both crude and standardised results should be presented when such adjustments are made.

In the past century, occupational differences in mortality have been well documented in studies using national or state mortality data. ${ }^{1-4}$ However, determining the relative contribution of direct occupational factors and indirect "lifestyle" or "social" factors to mortality excesses has been an important concern. In some situations this exercise is of debatable validity, since occupation, lifestyle, and social class are closely interdependent. Occupation itself may determine the lifestyle workers adopt or the social determinants of disease to which they are exposed. Social factors also usually play a role in determining occupation. Hence, the attempt to separate the effects of occupational and social factors may sometimes be misleading and obscure important causal relationships. There are, however, many occasions when it is appropriate to attempt to separate the effects of occupational and social factors, and it is this situation that we wish to consider here. In particular, we shall critically examine the methods that have previously been used for this task.

One such method, used by the British Registrar General, was to compare the mortality excess of men in a particular occupation with that of wives of men in the same occupation. Differences that also existed among wives were considered as having a social rather than occupational origin. Fox and Adelstein ${ }^{2}$ have criticised this approach and argued that it relies on assumptions that are not always satisfied. The first of these is that social factors have the same effect for women as for men. The second is that female mortality rates are not affected by occupational exposures. They criticised the second assumption, in particular, and demonstrated that married women's occupations tend to be similar to those of their husbands.

Fox and Adelstein ${ }^{2}$ suggested social class standardisation as an alternative to the comparison of male and female mortality, but cautioned that "the assumptions on which it relies and its robustness need to be evaluated as critically as the male-female comparison". Despite this caution, social class standardisation has been routinely used in several occupational studies. ${ }^{134}$

Social class standardisation relies on two assumptions which are analogous to those previously used in the male-female comparison. The first is that social factors have the same effect in all occupational groups belonging to the same social class; the second is 
that a comparison group selected from the same social class will be free from occupational exposures which cause the study disease. In this paper our intention is to critically examine this second assumption.

\section{Job-related factors and social factors}

When controlling for social class, one wants to avoid bias which could be introduced by differential distribution of risk factors associated with social class, such as diet, housing conditions, ethnicity, smoking habits, leisure time activity, etc. The problem, with social class standardisation, is to separate the influence of social factors and occupational factors by the choice of an appropriate comparison group.

For purposes of illustration, let us assume that the effects of various risk factors are additive. The total incidence of the disease of interest in the study group is then the sum of the incidence due to the exposure, the incidence due to social factors, and the incidence due to unknown background factors (either unmeasured or truly unknown). In most situations, the various factors will contribute to common causal pathways, and the overall incidence will depart from additivity of its components. However, analogous arguments apply to this more complex situation. The components of

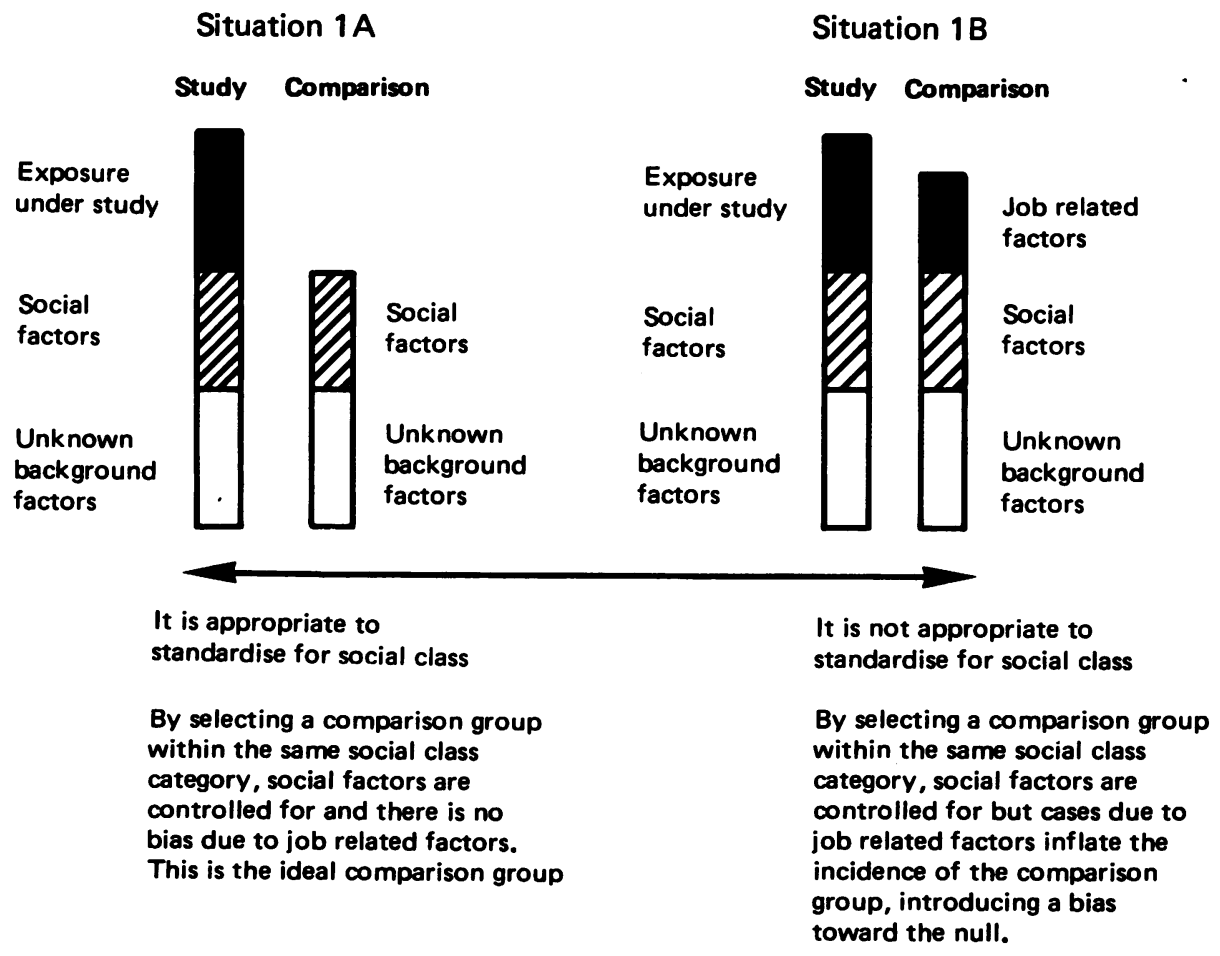

incidence in the study group are thus:

$$
I=I_{e}+I_{s}+I_{u}
$$

where, $I=$ incidence in the study group,

$I_{e}=$ incidence due to exposure,

$I_{s}=$ incidence due to social factors, and

$I_{u}=$ incidence due to unknown background factors.

The purpose of the comparison group is to estimate the quantity $I_{s}+I_{u}$, the incidence that would have occurred in the study group if it had not been exposed. In controlling for social class the researcher wants to insure that disease incidence due to social class factors $\left(I_{s}\right)$ and unknown background incidence $\left(I_{u}\right)$ are the same in the study group and the comparison group. Thus, any excess occurrence of the disease in the study group will be due solely to the occupational exposure under consideration.

The difficulty with this procedure is that job-related exposures associated with the disease under study may also occur in the same social class category. For example, many manual occupations involve exposure to toxic chemicals, fumes, and dust. In a comparison group that has job-related exposures, the incidence of the disease will be the sum of cases which are due to those exposures plus, as before, those which are due to social factors and those due to unknown factors. In

\section{Situation 1B}

It is not appropriate to

By selecting a comparison group controlled for but cases due to toward the null. 
this case the incidence in the comparison group is inflated by the contribution of other job-related factors, that is:

$$
I^{\prime}=I_{j r}{ }^{\prime}+I_{s}{ }^{\prime}+I_{u}{ }^{\prime}
$$

where $\mathbf{I}_{\mathrm{jr}}{ }^{\prime}=$ incidence due to job-related factors. The contributions of these various factors to disease occurrence are illustrated graphically in the figure. Situation A shows the contributions to total disease incidence in an ideal comparison group; all cases of the disease are caused by either social class factors or by unknown factors, and the incidence due to these two types of factors in the comparison group is equal to the incidence due to them in the study group. Confounding due to social factors would be perfectly controlled and all of the excess risk would be attributable to occupational exposure. Situation B illustrates a comparison group with additional cases caused by job-related factors. This comparison group would mask the true effect of exposure, and the effect measure would be biased toward the null. Figures $A$ and $B$ represent two extremes on a continuum of situations where at one end it is appropriate to control for social factors while at the other end it is misleading to do so.

\section{Examples}

To illustrate this problem, consider first a hypothetical study of non-malignant respiratory disease in cement plant workers exposed to dust. The investigators in such a study might want to control for extraneous risk factors by choosing a comparison group from among workers at a similar socioeconomic level, for example, other labourers. However, many labourers in construction, agriculture, road repair, and other industries are exposed to dusty conditions and might also be at elevated risk for respiratory disease. In this case the choice of other labourers as the comparison group would be given an artefactually low estimate of the effect of employment in a cement plant on workers' health.

Results from published studies illustrate similar problems. Milham's ${ }^{3}$ comparisons of the proportionate mortality of orchard farmers to that of two other groups, all farmers and all employed individuals, for lung cancer and machinery accidents are shown in the table. For machinery accidents, orchard farmers have a high proportionate mortality $(\mathrm{SPMR}=384)$ compared to all occupations, but the excess is eliminated when all farmers are used as the comparison group (SPMR $=99$ ). This is likely to be because all farmers are occupationally exposed to machinery, and not because of extraneous social factors. The excess mortality from machinery accidents among orchard farmers would be obscured in a study which compared mortality of several
Mortality in orchard farmers. White men, Washington State, USA, 1950-81.

\begin{tabular}{lllc}
\hline Cause of death & $\begin{array}{l}\text { Deaths } \\
\text { observed }\end{array}$ & SPMR* & SPMR** \\
\hline Machinery accidents & 13 & 384 & 99 \\
Lung cancer & 154 & 130 & 149 \\
\hline
\end{tabular}

* Standardised proportionate mortality ratio computed using all deaths, all occupations.

** Standardised proportionate mortality ratio computed using deaths in all farmers.

Source: derived from Milham ${ }^{3}$.

farming subgroups with that of all farmers. In contrast, the excess proportionate mortality for lung cancer increases slightly when all farmers, rather than all workers, are used as a comparison group (SPMR $=130$ for all occupations, 149 for all farmers). Most cases of lung cancer are caused by smoking, which is less prevalent among farmers. ${ }^{5}$ In this case, comparing the lung cancer mortality of orchard farmers to state mortality rates may be invalid since the general population rates may overestimate the "expected" lung cancer mortality of farmers. Thus, it may be more valid to compare the lung cancer mortality of orchard farmers with that of other farmers (who might be expected to have similar smoking prevalence). Hence, the adjustment seems useful and appropriate in this situation.

Milham notes these problems with stratification on farming, but attributes them to the stratification being too narrow. However, the narrowness of farming as an occupational category is clearly not the major issue, since stratification on the same occupational category apparently produced valid results for lung cancer but biased results for machinery accidents. The key issue is the relative contributions of social factors and jobrelated factors in producing the disease of interest in the occupational or social class category used as a comparison group.

\section{Discussion}

The presence of job-related exposures in the comparison group is always a possibility in occupational epidemiological studies. When the comparison group is the entire national population this problem is usually of little concern, since any such exposures would be expected to make only a small contribution to national incidence or mortality rates. However, when the comparison group is the social class to which the occupation of interest belongs, then this bias is of more concern. An additional problem in studies using national or state mortality data is that the comparison group contains the occupation of 
interest. Bias may thus occur if the comparison group is defined too narrowly, so that the study group forms a sizeable component of the comparison group. Both considerations would tend to produce a bias towards the null.

It should be emphasised, in particular, that broad occupational categories may not be the most desirable indicators of social class. For example, the broad category "farmers" can include both farm managers and farm labourers. This problem may be more severe for female workers, because women's jobs do not always reflect their educational level and family income. ${ }^{6}$ Clerical workers, for example, can have a broad spectrum of skills and responsibilities as well as a wide range of personal and family income. Such differences would not be adequately taken into account by a social class indicator based on crude occupational categories. Thus, stratification on occupational categories of this type may achieve only weak control over social factors but have great potential for bias due to job-related factors, as in the example of farming machinery accidents cited above. Useful social class scales based on detailed occupational information have been devised, ${ }^{7}$ but alternative indices, including scales based on a combination of occupation and education and/or income, are also valuable. ${ }^{89}$

These issues have been discussed here, and in previous publications, in the context of physical and chemical occupational hazards. The same principles should apply to studies of psychosocial occupational hazards (although few such studies have been conducted to date). For example, in a study of an occupation involving a particular psychosocial exposure, the investigator might be concerned about psychosocial exposures in the comparison group. The potential strength of such job-related psychosocial factors is demonstrated by the finding of Karasek $e t$ $a l,{ }^{10}$ which showed that occupational groups with high job demand and low job latitude have a higher frequency of CHD; these work characteristics were shown to be better predictors of disease risk than education, smoking, and overweight. For this reason, the bias due to the influence of job-related factors in a comparison group may outweigh the benefit of controlling for social factors through a social class indicator.

The potential problem of unmeasured job-related factors should also be considered in studies using internal comparison groups, such as unexposed workers in the industry under study, but are less likely to result in bias. In this case the focus is on the agent, rather than on employment in a particular occupation. The most appropriate solution in this context is to measure and adjust for exposure to those other factors.
The contribution of social class factors to the incidence of the disease under study will generally be greatest in lower social class groups since they have the highest rates for most disease outcomes. In occupational studies, the exposed group frequently belongs to that social class category simply because hazardous jobs are often encountered among manual workers.

However, the unintentional bias which may be introduced by controlling for social class is also more likely to be large in that circumstance, again because the lowest social groups usually have the most hazardous jobs and, thus, the greatest potential for job-related factors. The researcher must evaluate which one of these potential biases is more important to avoid. This decision can be made in the light of what is known about the presence of job-related factors in the comparison group and the contribution of social factors to incidence of the disease under study.

In summary, the method of social class standardisation proposed by Fox and Adelstein to separate the effects of occupation from those of social and lifestyle factors in occupational studies may suffer from a drawback similar to the practice it is intended to replace. The validity of this method depends upon the assumption that workers in the same social class as those being studied do not have excess mortality due to occupational risk factors. This assumption will not always be satisfied. Thus, although adjustment for social class should minimise bias due to social factors, it may introduce bias due to job-related factors. For this reason, adjustment for social class should be done only after careful consideration of the exposure and disease involved. When the adjustment is performed, it should be based upon an appropriate definition of social class, rather than on crude occupational category, and both the crude and standardised results should be presented.

We are grateful to Andy Rowland for helping to bring this issue to our attention and for his critical review of the manuscript. We also thank Steve Wing for his review and for many enlightening discussions on the sociological dimensions of health and disease.

\section{References}

${ }^{1}$ Office of Population Censuses and Surveys. Occupational mortality, 1970-1972. Decennial supplement. London: HMSO, 1978.

2 Fox AJ, Adelstein AM. Occupational mortality: work or way of life? J Epidemiol Comm Hlth 1978; 32: 73-8.

${ }^{3}$ Milham S. Improving occupational standardised proportionate mortality ratio analysis by social class stratification. Am J Epidemiol 1985; 3: 472-5. 
${ }^{4}$ Pearce NE, Howard JK. Occupation, social class and male cancer mortality in New Zealand, 1974-78. Int $J$ Epidemiol 1986; 15: 456-62.

-5 Pomrehn RR, Wallace RB, Burmeister L. Ischemic heart disease mortality in Iowa farmers: the influence of lifestyle. JAMA 1982; 248: 1073-6.

${ }^{6}$ Arber S. Social class, non-employment, and chronic illness: continuing the inequalities in health debate. $\mathrm{Br}$ Med J 1987; 294: 1069-73.

${ }^{7}$ Leete R, Fox J. Registrar General's social classes, origin and uses. Population Trends 1977; 8: 1-7.
${ }^{8}$ Abramson JH, Gofin J, Habib J, Pridan H, Gotin J. Indicators of social class; a comparative appraisal of measures for use in epidemiological studies. Soc Sci Med 1982; 16: 1739-46.

${ }^{9}$ Susser NW, Watson W, Hopper K. Theories and indices of social class. In Sociology and medicine New York: Oxford University Press, 1985.

${ }^{10}$ Karasek R, Baker D, Marxer F, Albhom A, Theorell T. Job decision latitude, job demands, and cardiovascular disease: a prospective study of Swedish men. Am J Public Health 1981; 71: 694-705.

Accepted for publication July 1987 